In order to apply the theorem to (13) let [note assumption (2)]

$$
\frac{|\phi(x, t)|}{|x|} \leq m_{0}=\text { constant }
$$

so that (13) becomes

$$
|x(t)| \leq|f(t)|+\int_{0}^{t}|h(t, \tau)| m_{0}|x(\tau)| d \tau .
$$

Inequality (14) is of the same form as (3). Therefore, the theorem can be used to get a bound on $x(t)$. A more customary problem is to find a sector for $\phi(x, t)$ of the following form:

$$
-c x \leq \phi(x, t) \leq+c x, \quad 0 \leq c=\text { constant }
$$

so that $|x(t)|$ does not exceed a given bound.

If in (1)

$$
h(t, \tau) \equiv h(t-\tau),
$$

pole shifting [2, pp. 382-385] modifies the sector in (15) to

$$
c_{1} x \leq \phi(x, t) \leq c_{2} x, \quad c_{1} \neq c_{2} .
$$

\section{Comments and Conclusions}

A simple comparison theorem has been discussed and various applications have been shown. With little expenditure, the theorem can be extended to a nonlinear integral equation of the following type:

$$
x(t)=f(t)+\int_{0}^{t} h(t, \tau)|x(\tau)|^{\alpha} d \tau
$$

assuming, for instance, that either

$$
\alpha \geq 0, \quad|f(t)|>0
$$

or

$$
\alpha \geq 1, \quad f(t) \text { an arbitrary continuous function. }
$$

The scope of the theorem is adequate for the requirements of various stability investigations. Admittedly, if it is desired to generalize the theorem regardless of any application, one can proceed to a more general comparison theorem as is suggested by Nohel [5], for example.

\section{REFERENCES}

[1] F. G. Tricomi, Integral Equations. New York: Interscience, 1967, pp. 10-15. [2] J. C. Hsu and A. U. Meier, Modern Control Principles and Applications. New York: McGraw-Hill, 1968.

[3] G. Sansone and R. Conti, Nonlinear Differential Equations. New York: Macmillan, 1964 , pp. 11.12 .

[4] A. Halany, Differential Equations. New York: Academic, 1966, pp. 7, 8.

5] J. A. Nohel, "Some problems in nonlinear integral equations," $B u l l$. . Amer. Math. Soc., vol. 68, pp. 323-329, July 1962 .

\section{Absolute Stability of NLTV Discrete Systems}

\section{S. RAMARAJAN AND S. N. RAO}

\begin{abstract}
A frequency domain stability criterion for nonlinear time-varying (NLTV) discrete systems with a separable time-varying nonlinearity is derived using the Lyapunov approach. It is shown that Szegö's [1] criterion for nonlinear time-invariant discrete systems and Chen's [4] criterion for linear time-varying discrete systems can be obtained as special cases of the criterion derived here.
\end{abstract}

Manuscript received April 14, 1971; revised July 20, 1971.

S. Ramarajan is with the Department of Electrical Engineering, Indian Institute of Seience, Bangalore, 12 , India.

S. N. Rao is with the Department of Mechanical Engineering, Indian Institute of Science, Bangalore 12, India.
The stability of nonlinear time-invariant (NLTI) discrete systems has been considered earlier by Szegö [1] using the Lyapunov technique, and similar results were also independently obtained by Jury and Lee [2] and by Tsypkin [3] via Popov's method. Chen [4] has considered linear time-varying (LTV) discrete systems and derived a stability criterion. In this correspondence nonlinear time-varying (NLTV) discrete systems with a separable time-varying nonlinearity are considered, and a frequency domain stability criterion is derived using Lyapunov's method. It is also shown that Szegö's [1] and Chen's [4] criteria can be obtained as special cases from the criterion derived here.

Consider the system given by

$$
\begin{aligned}
x(n+1) & =A x(n)-d \psi(\sigma, n) \\
\sigma(n) & =2 b^{\prime} x(n)
\end{aligned}
$$

where the time-varying nonlinearity $\psi(\sigma, n)$ is assumed to be separable and can be written as $\psi(\sigma, n)=k(n) \phi(\sigma), \boldsymbol{A}$ is a constant square matrix, $d$ and $b$ are constant vectors, and the prime denotes matrix transpose. The open-loop pulse transfer function is given by

$$
W(z)=2 b^{\prime}(z I-A)^{-1} d .
$$

Let

$$
\begin{gathered}
0<k(n) \leq L<\infty \\
\Delta k(n-1)=k(n)-k(n-1) .
\end{gathered}
$$

Define $k^{*}(n)=k(n) / L$ so that (3) becomes

$$
\begin{aligned}
0 & <k^{*}(n) \leq 1 \\
\Delta k^{*}(n-1) & =k^{*}(n)-k^{*}(n-1) .
\end{aligned}
$$

Also let us assume that the nonlinearity $\phi(\sigma)$ satisfies the following two conditions: namely,

$$
0<\sigma \phi(\sigma) \leq K \sigma^{2}, \quad \sigma \neq 0, \quad \phi(0)=0, \quad K<\infty
$$

and

$$
0<\frac{d \phi(\sigma)}{d \sigma}<\mu
$$

Define $F_{\min }$ a measure [5] of the nonlinearity, as

$$
F_{\min }=\inf _{\sigma}\left[\frac{\sigma \phi(\sigma)}{\int_{0}^{\sigma} \phi(\rho) d \rho}\right]
$$

It can easily be seen that for linear cases $F_{\min }=2$ and for monotonic nonlinearities $F_{\min } \geq 1$.

Under condition (6) we have [1],[6]

$$
\begin{aligned}
\beta \int_{\sigma(n)}^{\sigma(n+1)} \phi(\rho) d \rho \leq \beta \phi[\sigma(n)][\sigma(n+1)-\sigma(n)] & \\
& +\frac{\mu|\beta|}{2}[\sigma(n+1)-\sigma(n)]^{2}
\end{aligned}
$$

where $\beta$ is a real number. If $\beta$ is restricted to be a positive real number, then (8) is true [3] for $-\infty<d \phi(\sigma) / d \sigma<\mu$. On the other hand, if $\beta$ is restricted to be a negative real number, then (8) holds for $-\mu<d \phi / d \sigma<\infty$.

The system (1) can also be written as

$$
\begin{aligned}
x(n+1) & =A x(n)-a k^{*}(n) \phi[\sigma(n)] \\
\sigma(n) & =2 b^{\prime} x(n)
\end{aligned}
$$

where $a=d L$ and the new open-loop pulse transfer function becomes

$$
W^{*}(z)=2 b^{\prime}(z I-A)^{-1} a=L W(z) .
$$


The following theorem gives the stability criterion for system (1).

Theorem: Consider the system (1) where $A$ is stable and the linear part is completely controllable and completely observable. Let the linearized system be stable for all linear gains in the range $(0, K L\}$. Then the equilibrium $x(n)=x(n+1)=0$ is asymptotically absolutely stable if there exist constants $\delta, \theta$, and $\lambda$ such that

1)

$$
\begin{aligned}
\delta\left[\frac{\lambda}{L K}+\operatorname{Re} W(z)\right] & +\frac{\theta}{L K}+\operatorname{Re}\{[\theta \pm(z-1)] W(z)\} \\
& -\frac{\mu L}{2}|(z-1) W(z)|^{2} \geq 0, \quad \forall|z|=1 ;
\end{aligned}
$$

2) $\left(\frac{\theta}{K}+\frac{\delta \lambda}{K} \pm 2 \xi\right)>0, \quad \theta \geq 0, \quad \delta>0, \quad 0 \leq \lambda \leq 1$,

$$
\xi=a^{\prime} b \mp \mu\left(a^{\prime} b\right)^{2}
$$

3) $\pm \Delta k(n-1) \leq \delta F_{\min } k(n)\left[1-\frac{\lambda k(n)}{L}\right]$.

Proof: Consider the Lyapunov function

$$
V(n)=x^{\prime}(n) H x(n)+\beta k^{*}(n-1) \int_{0}^{\sigma(n)} \phi(\rho) d \rho
$$

where $H$ is a symmetric positive-definite matrix and $\beta$ is a real constant. The $V$ difference of (11) along the solutions of system (9) is

$$
\begin{aligned}
\Delta V(n)= & x^{\prime}(n+1) H x(n+1)-x^{\prime}(n) H x(n) \\
& +\beta\left[k^{*}(n) \int_{0}^{\sigma(n+1)} \phi(\rho) d \rho-k^{*}(n-1) \int_{0}^{\sigma(n)} \phi(\rho) d \rho\right] \\
= & x^{\prime}(n)\left[A^{\prime} H A-H\right] x(n)-2 \phi[\sigma(n)] k^{*}(n) x^{\prime}(n) A^{\prime} H a \\
& +\phi^{2}[\sigma(n)] k^{* 2}(n) a^{\prime} H a \\
& +\beta\left[k^{*}(n) \int_{0}^{\sigma(n+1)} \phi(\rho) d \rho-k^{*}(n-1) \int_{0}^{\sigma(n)} \phi(\rho) d \rho\right] .
\end{aligned}
$$

The last term in (12) can be written as

$$
\begin{aligned}
& \boldsymbol{\beta}\left[k^{*}(n) \int_{0}^{\sigma(n+1)} \phi(\rho) d \rho-k^{*}(n-1) \int_{0}^{\sigma(n)} \phi(\rho) d \rho\right] \\
& =\beta\left[\Delta k^{*}(n-1) \int_{0}^{\sigma(n)} \phi(\rho) d \rho+k^{*}(n) \int_{\sigma(n)}^{\sigma(n+1)} \phi(\rho) d \rho\right] .
\end{aligned}
$$

Using (4) and (8), the second term in the right-hand side of (13) can be written as

$$
\begin{array}{r}
\beta k^{*}(n) \int_{\sigma(n)}^{\sigma(n+1)} \phi(\rho) d \rho \leq \beta k^{*}(n) \phi[\sigma(n)][\sigma(n+1)-\sigma(n)] \\
+\frac{\mu|\beta|}{2}[\sigma(n+1)-\sigma(n)]^{2} .
\end{array}
$$

By adding to and subtracting from the first term in the right-hand side of (13), the quantity

$$
\left\{|\beta| \delta k^{*}(n) \sigma(n) \phi[\sigma(n)]+|\beta| \tau k^{* 2}(n) \sigma(n) \phi[\sigma(n)]\right\}
$$

where $\delta>0$ and $0 \leq \tau / \delta \leq 1$, we get (after simplifieation)

$\beta \Delta k^{*}(n-1) \int_{0}^{\sigma(n)} \phi(\rho) d \rho=|\beta| \sigma(n) \phi[\sigma(n)]\left\{\Delta k^{*}(n-1)(\operatorname{sgn} \beta)\right.$

$$
\begin{aligned}
& \left.\cdot\left(\frac{\int_{0}^{\sigma(n)} \phi(\rho) d \rho}{\sigma(n) \phi[\sigma(n)]}\right)-\delta k^{*}(n)\left(1-\frac{\tau}{\delta} k^{*}(n)\right)\right\} \\
& +|\beta| \delta k^{*}(n) \sigma(n) \phi[\sigma(n)]-|\beta| \tau k^{* 2}(n) \sigma(n) \phi[\sigma(n)] .
\end{aligned}
$$

The first term in the right-hand side of the preceding equation is nonpositive if

$$
\Delta k^{*}(n-1) \operatorname{sgn} \beta \leq F_{\min } \delta k^{*}(n)\left(1-\frac{\tau}{\delta} k^{*}(n)\right) .
$$

Hence, using (5) we get

$$
\begin{aligned}
\beta \Delta k^{*}(n-1) \int_{0}^{\sigma(n)} \phi(\rho) d \rho \leq|\beta| \delta k^{*}(n) \sigma(n) \phi[\sigma(n)] \\
\\
-\frac{|\beta| \tau}{K} k^{* 2}(n) \phi^{2}[\sigma(n)] .
\end{aligned}
$$

Using (13), (14), and (16) in (12), we obtain

$$
\begin{aligned}
\Delta V(n) \leq & x^{\prime}(n)\left[A^{\prime} H A-H\right] x(n)-2 \phi[\sigma(n)] k^{*}(n) x^{\prime}(n) A^{\prime} H a \\
& +\phi^{2}[\sigma(n)] k^{* 2}(n) a^{\prime} H a+\beta k^{*}(n) \phi[\sigma(n)][\sigma(n+1) \\
& -\sigma(n)]+\frac{\mu|\beta|}{2}[\sigma(n+1)-\sigma(n)]^{2} \\
& +|\beta| \delta k^{*}(n) \phi[\sigma(n)] \sigma(n)-\frac{|\beta| \tau}{K} k^{* 2}(n) \phi^{2}[\sigma(n)]
\end{aligned}
$$

Substituting for $\sigma(n)$ and $\sigma(n+1)$ and adding and subtracting

$$
\alpha \phi[\sigma(n)] k^{*}(n)\left[\sigma(n)-\frac{\phi[\sigma(n)] k^{*}(n)}{K}\right]
$$

where $\alpha \geq 0$, from the right-hand side of the preceding inequality we have

$$
\begin{aligned}
\Delta V(n) \leq & x^{\prime}(n)\left[A^{\prime} H A-H+r^{\prime}\right] x(n) \\
& -2 k^{*}(n) \phi[\sigma(n)] x^{\prime}(n)\left[A^{\prime} H a-\alpha b-|\beta| \delta b-\beta c\right] \\
& -k^{* 2}(n) \phi^{2}[\sigma(n)]\left[\frac{\alpha}{K}+\frac{|\beta| \tau}{K}+2 \beta \xi-a^{\prime} H a\right] \\
& -\alpha \phi[\sigma(n)] k^{*}(n)\left[\sigma(n)-\frac{\phi[\sigma(n)] k^{*}(n)}{K}\right]
\end{aligned}
$$

where $r, c$, and $\xi$ are defined by

$$
\begin{aligned}
& r=\sqrt{2 \mu|\beta|}(A-I)^{\prime} b \\
& c=-\left(2 \mu b^{\prime} a \operatorname{sgn} \beta-1\right)(A-I)^{\prime} b \\
& \xi=a^{\prime} b-\mu\left(a^{\prime} b\right)^{2} \operatorname{sgn} \beta .
\end{aligned}
$$

Now let us consider a slightly modified version of the lemma due to Szegö [1], [7].

Lemma: Necessary and sufficient conditions for the existence of a real scalar $\gamma$, a real vector $q$, and a real matrix $H=H^{\prime}>0$ such that

$$
\begin{aligned}
& A^{\prime} H A-H+r^{\prime}+q q^{\prime}=0 \\
& A^{\prime} H a-\alpha b-|\beta| \delta b-\beta c=\gamma q \\
& 2 \beta \xi+\alpha / K+|\beta| \tau / K-a^{\prime} H a=\gamma^{2}>0
\end{aligned}
$$

are

$$
\text { 1) } \begin{aligned}
\frac{\alpha}{K}+\frac{|\beta| \tau}{K}+2 \beta \xi+2 \operatorname{Re}\left\{(\alpha b+|\beta| \delta b+\beta c)^{\prime}(z I-A)^{-1} a\right\} \\
-\left|r^{\prime}(z I-A)^{-1} a\right|^{2} \geq 0, \quad \forall|z|=1 ;
\end{aligned}
$$


2) $\frac{\alpha}{K}+\frac{|\beta| \tau}{K}+2 \beta \xi>0$

Under the conditions of (20), (17) becomes

$$
\Delta V(n) \leq-\left[q^{\prime} x(n)+\gamma \phi[\sigma(n)] k^{*}(n)\right]^{2} .
$$

Following the arguments of Szego [1], we can show that $\Delta V(n)=0$ if and only if $x(n+1)=x(n)=0$ (i.e., at the equilibrium point) and $\Delta V(n)<0$ for $x(n) \neq 0$. Also, since the right-hand side of $(21)$ is independent of $\beta$, by an argument similar to that of Szegö [1], we see that $V$ is positive definite, even when $\beta$ is negative.

Thus the system (9) and, hence, the system (1) are asymptotically stable if the conditions of (20) are satisfied for some $\alpha \geq 0$ along with (15). Substituting for $\xi$, $c$, and $r$ in (20), we get

1) $\frac{\alpha}{K}+\frac{|\beta| \tau}{K}+\operatorname{Re}\left\{[\alpha+|\beta| \delta+\beta(z-1)] W^{*}(z)\right\}$

$$
-\frac{\mu|\beta|}{2}\left|(z-1) W^{*}(z)\right|^{2} \geq 0, \quad \forall|z|=1 ;
$$

2) $\frac{\alpha}{K}+\frac{|\beta| \tau}{K}+2 \beta \xi>0, \quad \alpha \geq 0$.

Now replacing $W^{*}(z)$ by $L W(z)$ and dividing throughout by $|\beta|$ and denoting $\alpha /|\beta|=\theta \geq 0$, (22) becomes

1) $\frac{\theta}{L K}+\frac{\tau}{L K}+\operatorname{Re}\{[\theta+\delta+(z-1) \operatorname{sgn} \beta] W(z)\}-$

$$
-\frac{\mu L}{2}|(z-1) W(z)|^{2} \geq 0, \quad \forall|z|=1 ;
$$

2) $\theta \geq 0, \frac{\theta}{K}+\frac{\tau}{K}+2 \xi \operatorname{sgn} \beta>0$;

and correspondingly, (15) becomes

$$
\Delta k(n-1) \operatorname{sgn} \beta \leq F_{\min } \delta k(n)\left[1-\frac{\tau}{\delta} \frac{k(n)}{L}\right] .
$$

Putting $\tau / \delta=\lambda,(23)$ and (24) can be written as

$$
\text { 1) } \begin{aligned}
\delta\left[\frac{\lambda}{L K}\right. & +\operatorname{Re} W(z)]+\frac{\theta}{L K} \\
& +\operatorname{Re}\{[\theta+(z-1) \operatorname{sgn} \beta] W(z)\} \\
& -\frac{\mu L}{2}|(z-1) W(z)|^{2} \geq 0, \quad \forall|z|=1 ;
\end{aligned}
$$

2) $\left[\frac{\theta}{K}+\frac{\delta \lambda}{K}+2 \xi \operatorname{sgn} \beta\right]>0, \quad \forall \theta \geq 0, \quad \delta>0, \quad 0 \leq \lambda \leq 1$;

$$
\text { 3) } \Delta k(n-1) \operatorname{sgn} \beta \leq \delta F_{\min } k(n)\left[1-\frac{\lambda k(n)}{\mathrm{L}}\right] \text {; }
$$

which are nothing but $1-3$ of the theorem with $\operatorname{sgn} \beta= \pm 1$ (i.e., $\beta$ either positive or negative), and hence the proof.

\section{Nonlinear Time-Invariant Case}

Szegö's [1] criterion for the NLTI case can easily be obtained by putting $\delta=0, L=1, K=k$, and $\theta=(\gamma+\beta) /|\beta|$ in (25).

\section{Linear Time-Varying Case}

For the linear case, $F_{\min }=2$. Taking $\phi(\sigma)=K \sigma$, we see that $\mu=K$. Let $K L=k$, so that $k(n) \phi(\sigma) \sigma=k(n) K \sigma^{2} \leq K L \sigma^{2}$ or $\leq k \sigma^{2}$. Now we get the following conditions:
1) $\frac{\theta}{k}+\delta\left[\frac{\lambda}{k}+\operatorname{Re} W(z)\right]+\operatorname{Re}\{[\theta \pm(z-1)] W(z)\}$

$$
-\frac{k}{2}|(z-1) W(z)|^{2} \geq 0, \quad|z|=1
$$

2) $\quad \frac{\theta+\delta \lambda}{K} \pm 2 \xi>0, \quad \theta \geq 0, \quad \delta>0, \quad 0 \leq \lambda \leq 1$

3) $\quad \pm \Delta k(n-1) \leq 2 \delta k(n)\left[1-\frac{\lambda k(n)}{L}\right]$.

One way of eliminating condition 2 is to allow $K \rightarrow 0$ and $L \rightarrow \infty$ such that $K L=k$ (a constant) so that condition 2 is obviously satisfied, since the first term becomes large as $K \rightarrow 0$ and $\xi \rightarrow\left(a^{\prime} b\right)$, which is finite. So the criterion reduces to (with $k(n)=K k(n)$ so that $0<k(n) \leq k)$ the following:

1)

$$
\begin{aligned}
\frac{\theta}{k}+\delta\left[\frac{\lambda}{k}+\operatorname{Re} W(z)\right]+\operatorname{Re}\{[\theta \pm(z-1)] W(z)\} \\
-\frac{k}{2}|(z-1) W(z)|^{2} \geq 0, \quad \forall|z|=1 ;
\end{aligned}
$$

2) $\quad \pm \Delta k(n-1) \leq 2 \delta k(n)\left[1-\frac{\lambda k(n)}{k}\right]$,

$$
\theta \geq 0, \quad \delta>0, \quad 0 \leq \lambda \leq 1, \quad 0<k(n) \leq k .
$$

Chen's criterion [4] for LTV cases can be obtained by putting $\theta=0, \lambda=1, \delta=1 / q$, and using only the positive sign in (26).

\section{REFERENCES}

[1] G. P. Szegö, "On the absolute stability of sampled-data systems," Proc.

[2] F. I. Jury and B. W. Lee, "On the stability of a certain class of nonlinear sampled-data systems," IEEL Trans. A utomat. Contr., vol, AC-9, pp. 51-61,

[3] Ya. Z. Tsypkin, "A bsolute stability of nonlinear automatic sampled-data systems," Automat. Remote Contr. (USSR), vol. 25, pp. 1030-1036, 1964.

[1] C. T. Chen, "On the stability of sampled-data feedback systems with timevarying gain," IEEE Trans. Automat. Contr. (Corresp.), vol. AC-11, pp. $624-625$, July 1966

[5] K. S. Narendra and J. H. Taylor, "Liapunov functions for nonlinear time-

[6] Varying systems, Inform. Contr.. vol. 12 , pp. 378-393, 1968.

. B. Pearson, $J r$, and $J$. G. Gibson, "On the asymptotic stability of a class of saturating sampled

[7] G. P. Szegö and R. E. Kalman. "Absolute stability of a system of finito difference equations," $C$. $R$. Acad. Sci., vol. 256, pp. 388-390, 1963.

\section{Stability Criteria for a Class of Multiplicative Time-Varying Nonlinear Systems}

\section{MIN-YEN WU}

Abstract-Sufficient conditions for the stability of a class of multiplicative time-varying as well as time-invariant nonlinear systems are presented.

The stability problem of feedback systems with multiplicative nonlinearities has been of current interest, and several results are available in the literature [1], [2]. Recently Zames' passivity theorem [3] has been applied to obtain sufficient conditions in the frequency domain for the stability of a class of multiplicative time-invariant nonlinear systems [1]. In this correspondence we present sufficient conditions for the stability for the same system shown in [1, fig. 1), but with a broader class of linear subsystems and nonlinearities.

Manuscript received July 21, 1971; revised September 7, 1971.

The author is with the Department of Electrical Engineering, University of Colorado, Boulder, Colo. 80302. 